\title{
Awareness Among Students and Staff on Occupational Safety and Health (OSH) in Universiti Teknologi Malaysia
}

\author{
Faiqah Binti Ramli ${ }^{* 1}$, Siti Nabila Binti Mokhtar ${ }^{1}$, Mohd Haizal Bin Jamaluddin ${ }^{1}$, \\ Muhammad Noor Bin Harun ${ }^{1}$, Muhammad Aizi Bin Mat Salim ${ }^{1}$, Muhammad Nizam \\ Bin Lokman ${ }^{1}$, Abdul Rashid Bin Zailan ${ }^{1}$, Norhidayah Binti Md. Yunus ${ }^{1}$, \\ Nurshaidatul Hidayah Binti Mohd Nor ${ }^{1}$
}

${ }^{1}$ Kolej Rahman Putra (KRP), Universiti Teknologi Malaysia (UTM), Campus Johor, 81310 Johor Bahru, Johor, Malaysia.

*Corresponding author. Email: faiqah2@gmail.com

\begin{abstract}
Laboratory plays important role to ensure efficient scientific work and promotes safety work environment. Nevertheless, there are an increased numbers of reported laboratory accidents led to numerous catastrophic calamities such as injuries and fatalities. Therefore, it is important to access current research status and trends that meets safety research environment prior to laboratory standard. Occupational Safety and Health (OSH) is a performance standard designed to protect employees working in both laboratory and industrial area. This act has been implemented in academic community and essentially replaced safety education among students to develop strong safety ethics. This study is designed to access factors effecting consciousness among students and laboratory staff in Universiti Teknologi Malaysia such as safety policy, safety procedure, safety training, safety tools and equipment, OSH committee, commitment and attitude and working environment. Findings conclude that the respondents are considered to possess high level of consciousness toward OSH with overall mean grade of 4.20. The overall mean grade of staff (4.26) is higher than students (4.14).
\end{abstract}

Keywords: Occupational Safety and Health; laboratory; consciousness

\section{INTRODUCTION}

Potential health risk at work such as workplace injury and illness has been long perceived since middle ages. Although safety and health are closely related, they presumably carry different implication. Safety can be defined as injurycausing situation whereas health concerns with disease-causing situations. In addition, safety may also associated with hazards to human well beings as a result if improper management of environmental safety [1]. For example, occupational injuries account for huge distresses in terms of substantial economic costs, major injuries and even fatalities [2]. Study reported by [3] demonstrates that average occupational fatalities reported worldwide is about two millions each year. Laboratory users such as researchers, graduate students and laboratory assistants are exposed to a great risk of hazards and their health may be endangered if not properly trained or managed [4].

Scientific research or academic laboratories often considered of having lower risk of injuries than industrial scale laboratories [5]. Nevertheless, it is worth noting that there have been numerous reports of laboratory calamities occurred in academic communities that resulted in serious injury of fatalities. In 2013, a chemistry lab in Universiti Teknologi Mara, Shah Alam was destroyed by a fire while students and laboratory assistant were carrying out experiments. Recently, 
13 students in Bayan Lepas were reported to accidentally inhaled iodine gas as a result of poor air ventilation in laboratory workplace. Similarly the number of laboratory mishaps reported around the world is increasing. In the U.S, there were 49 fatalities reported as a result of 34 laboratory accidents. These catastrophes spark the need to search for the root causes and implement a risk management system within universities to avoid any unfortunate events [5].

There are multiple hazardous conditions in laboratory as a result of wide variety chemical usage [6]. Thus, in 1990, Occupational Safety and Health $(\mathrm{OSH})$ introduced a standard regulation to protect employees and students working in laboratories. The implementation of the regulation brought positive impact in laboratories working environment particularly among higher learning institution. Following mandate to implement the regulations, academic institution has been following the requirements such as having a chemical hygiene plan, trainings, laboratory designs and various safety programs purposely to prepare students with the broad safety ethics [7]. OSH covers several subjects that resulted to dramatic changes in Lab Standard such as Chemical Hygiene Plan (CHP), Chemical Hygiene Officer, Safety Data Sheet (SDS), Safety Information Training, Standard Operation Procedure (SOP), Personal Protective Equipment (PPE), Designated Area, Medical Consultation, and Safety Resources [8].

Contrary to popular belief, despite the implementation of $\mathrm{OSH}$ regulation in universities laboratories in 1990s, academic institutions have experienced a great number of reported laboratory adverse incidents. Majority incidents reported in laboratories could be associated to poor or inadequate safety management [9]. Hence, there is a little doubt that academic institutions have not been implementing $\mathrm{OSH}$ regulations to manage risk in experimental processes [5]. In response, this study aimed to accessed factors affecting OSH awareness among laboratory users in University Teknologi Malaysia. To achieve the objective, we use quantitative technique through a survey from a sample of working adults and college students. Pearson correlation was employed to determine the relationship between respondents' awareness towards work safety. The present work is helpful for researcher to have better understanding of safety awareness and management among UTM laboratories employees and students.

\section{LITERATURE REVIEW}

According to previous research done by [5] work injuries can be divided into two categories; academic research and large scale industrial processes. A study conducted by [10] discovers satisfactory level of consciousness of OSH among the estate's rubber tappers. However, the level of non-compliance with $\mathrm{OSH}$ and procedure was lower than average. The findings imply the need to take actions to overcome the inadequate training or awareness among the employees. Similarly, a study was carried out to determine the factors that could contribute to unfortunate mishaps at construction sites. The findings reveals that the main factor contributed to workplace injury at construction site was lack of awareness and poor site management [4]. Safety management program and traditional occupational safety audits to ensure regulations compliance are essential steps to reduce the likelihood of reoccurring unfortunate incidents [11].

From educational sector point of view, although it is be true that numbers of hazards are practically smaller in universities which contributed to lower associated laboratory incidents as compared to large-scale process industry, statistics have proven that a better risk management should be applied within academic teaching and experimental labs. 
There are several challenges in applying safety management program in university level such as relatively young and inexperienced employees [12]. Hence, the verification of experience and training at university becomes very critical. In addition, lack of safety culture among laboratory users could be the factor to several reported incidents. It is worth noting that commitment from the dean's office as well safety officers play important role in order to make sure risk of unfortunate events is reduced. Furthermore, it seems to be necessary to carry out risk assessment or ad-hock expert evaluation from time to time to identify potential hazards within laboratories and to understand prevention method of the risk.

A survey was carried out survey to access the level of occupational safety and health awareness among UTM employees [10]. The awarenessraising safety measure is policy, procedure, training, tools and equipment. The outcome indicates that UTM has an average level of OSH compliance of 3.54 and the dominant factor that complies with OSH 1994 is commitment and attitude, safety training and security procedure.

\section{METHODOLOGY/MATERIALS}

The survey used in this study were retrieved from [13] and [10] from a sample of working adults (25 years old or older) and undergraduate students at laboratories and engineering workshops in UTM. The study was collected through a survey at five different schools that were identified to have higher risk of workplace injury: School of Science, School of Mechanical Engineering, School of Civil Engineering, School of Electrical Engineering and School of Chemical and Energy Engineering. The survey was conducted during academic study of 2019.

The self-administered questionnaires can be divided into two parts. Part A was developed to according to literature review to access the demographic and occupational data of respondents. The second set of survey was specifically designed to access the level of safety awareness among UTM students and laboratory assistants. The questionnaire focuses on factors that could contribute to lack of OSH awareness among laboratory users. Seven factors were chosen in this study according to literature review includes safety policy, safety procedure, safety training, safety tools and equipment, safety committee, commitment and attitude as well as environment. In the present work, the analysis is performed using bibliometric Microsoft Excel to access the frequency, percentage and mean.

\section{RESULTS AND DISCUSSION}

The questionnaires for this study were obtained and modified from research conducted by [13] and [10]. The study was conducted in University Teknologi Malaysia. The respondents comprise of staffs and students in faculties with high risk of equipment or substances namely School of Science, School of Mechanical Engineering, School of Civil Engineering, School of Electrical Engineering, and the School of Chemical Engineering and Natural Resources. There are a total of 50 respondents where 25 respondents are students and the other 25 respondents are laboratory staffs. Hence, the ratio of students to staffs is $1: 1$. The respondents were categorized into three categories which are which are age, gender, education level. Seven factors were selected in this study which was believed to be the core factor of awareness; safety policy, procedure, training, tools and equipment, committee, commitment and behavior, and operative environment.

Table 1 shows that overall mean of consciousness among students and staffs in University Teknologi Malaysia are $4.20(>4.00)$ which indicates that the staffs and students have great level of awareness in $\mathrm{OSH}$. The outcome of mean grade for the factors of safety policy, procedure, training, tools and equipment, committee, commitment and behavior, and operative environment are tabulated in the 
Table 1 and Figure 1. Safety policy has the highest mean for the students and staffs which is 4.40 and 4.60 , respectively. These findings indicate that most laboratory users in UTM have high awareness on safety policy.

Table 1: Mean Grade for Factors that Contributes to the Level of Consciousness on $\mathrm{OSH}$ at Laboratory

\begin{tabular}{|c|c|c|c|}
\hline \multirow{2}{*}{ Factors } & \multicolumn{3}{|c|}{ Position } \\
\cline { 2 - 4 } & Staff & Students & Overall \\
\hline $\begin{array}{c}\text { Safety } \\
\text { policy }\end{array}$ & 4.60 & 4.40 & 4.50 \\
\hline $\begin{array}{c}\text { Safety } \\
\text { procedure }\end{array}$ & 3.92 & 4.00 & 3.96 \\
\hline $\begin{array}{c}\text { Safety } \\
\text { training }\end{array}$ & 4.24 & 4.36 & 4.31 \\
\hline $\begin{array}{c}\text { Safety tools } \\
\text { and } \\
\text { equipment }\end{array}$ & 4.32 & 4.32 & 4.32 \\
\hline $\begin{array}{c}\text { Safety } \\
\text { committee }\end{array}$ & 3.72 & 3.36 & 3.54 \\
\hline $\begin{array}{c}\text { Commitment } \\
\text { and attitude }\end{array}$ & 4.44 & 4.24 & 4.34 \\
\hline Environment & 4.56 & 4.28 & 4.42 \\
\hline Total & 4.26 & 4.14 & 4.20 \\
\hline
\end{tabular}

Nevertheless, as tabulated in Table 1, safety committee factor showed the lowest of mean grade for the students and staff which is 3.36 and 3.72, respectively. Safety committee recorded the lowest mean grade of 3.54 . $53 \%$ of the respondents admitted that the safety committee did not execute their tasks excellently and $65 \%$ of them mentioned that the safety committee has not carried out training activity frequently. Moreover, 55\% of the respondents did not notice the fact that the lecturers and technicians from the campus are also a part of safety OSH committee.

These findings should be a wakeup call for the safety committee to deliver safety meetings in regular basis and one to one discussion about safety with laboratories users. A one to one meeting could be a good approach as the respondents may discuss any safety-related issues with regards to their experimental protocols or the working environment, at the same time providing them a great opportunity to recognize safety committee members and their purposes. In addition, the safety committee should firsthandedly host safety workshops regularly for laboratory users to increase hazard awareness and $\mathrm{OSH}$ regulations in handling hazardous chemicals and experimental tools. Furthermore, direct one to one safety demonstrations during the workshops could definitely enhance safe environment workplace and simultaneously reduce hazard risk as the laboratories users need to be reminded once in a while of their hazard working area.

In contrary, safety policy has recorded highest mean grade of 4.50 as compared to other factors. The highest mean grade of safety policy suggests that the students and staff are more familiar with regulation and policies implemented by safety committee. Safety policies are important independent factors in providing a proper functioning society. It is important to note that the good awareness of safety policy shows that although the respondents did not recognize the member or function of safety committee, but they are still able to identify potential hazards and understand the risk of experimental tools in laboratories working environment. A possible explanation of this situation is the policy and guidelines of occupational safety and health, (Occupational Safety and Health 1994), and its regulations are provided by the department for every first entry of the students or any laboratory users upon registering the laboratories. Furthermore, the policies and regulations are often publicly shown in a safety area in each laboratory or in area with high risk of potential hazard to remind the laboratory users. A preliminary hazard checklist and necessary assessment tool is also provided to every laboratory users during training programs to increase awareness and simultaneously reduce hazard risk in laboratories. Over the years, these techniques have been proven to be efficient to provide clear instructions and require minimum level of resources. 


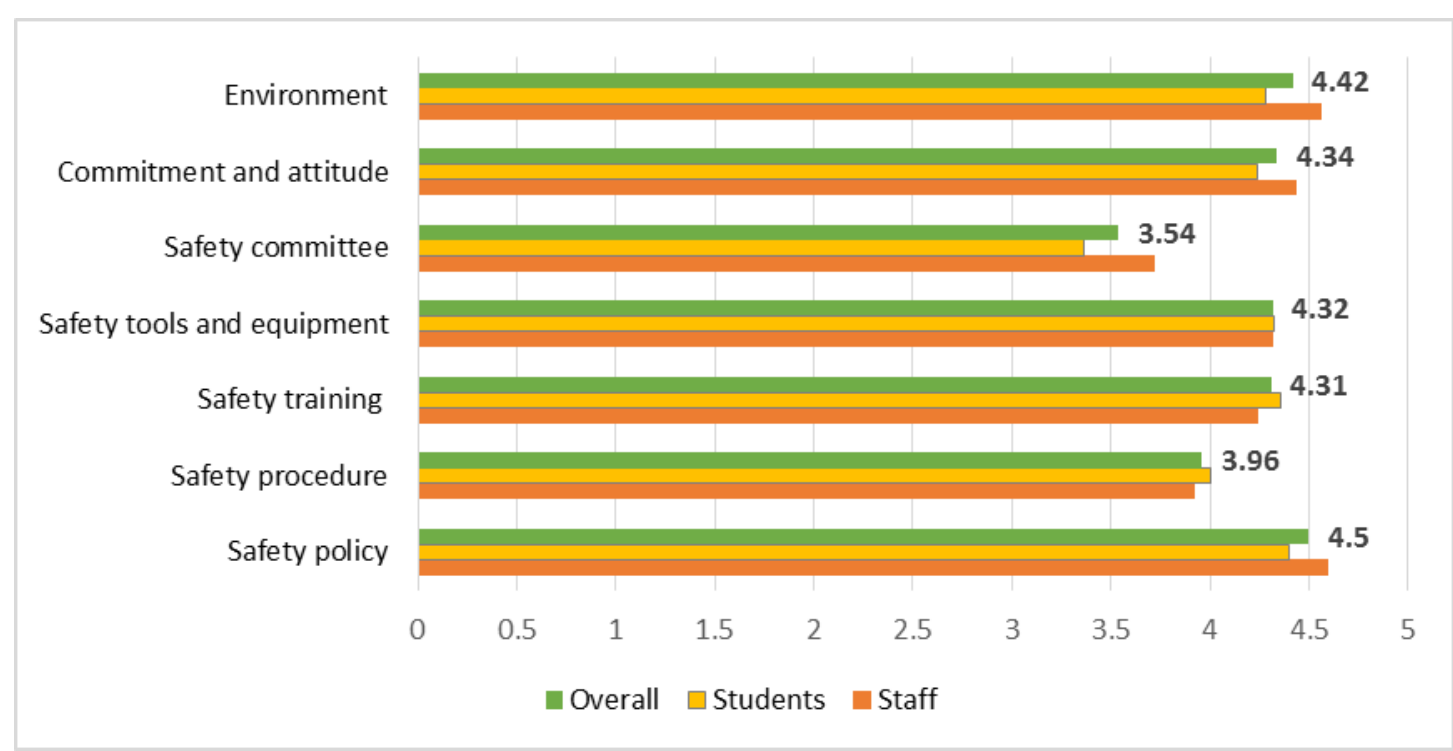

Figure 1: Factors contribute to the level of consciousness on OSH at laboratory

As depicted in Figure 1, UTM staffs have higher level of awareness toward OSH (total mean grade of 4.26) as compared to the students in UTM (total mean grade 4.14). A possible explanation for this finding is that UTM staffs are more aware of OSH regulations especially in four major factors namely safety policy, safety committee, commitment and attitude as well as environment. This is most likely due to the staffs are often required to attend trainings and seminars to improve their knowledge on OSH regulations. In summary, considering all the seven factors chosen, both staffs and students have the high level of awareness toward OSH in UTM.

Though the awareness of staffs and students on OSH is fairly good, however, it can be better by improving and making some adjustments to the bureau involved in OSH. Technically, a university is a large organization which involves a lot of management bureaucracy. Amendments towards the system could provide an efficient and safe working environment. Hence, the university management is encouraged to provide necessary resources to ensure safe operations at working place. This requires strong commitment among the university administration to have regular meeting with safety committees from each school and departments which hopefully to secure safety workplace beyond compliance.

Furthermore, besides the vigorous commitment from the university leadership, it is highly recommended for each safety personnel to perform risk assessment competencies through adapted hazards laboratory. The assessment could be conducted by safety personnel to identify potential hazard and the risk attributed. The staffs or students with less experience in handling risk assessment should participate in hazard identification training conducted by university leadership in order to control risk production in laboratories. Additionally, safety competence could be improved by developing a universal infrastructure and human resources whom focused on certain job scope within department with appropriate language usage and training inclusive. By implementing this advance policies, safety programs and safety campaigns could consequently increase awareness on OSH among UTM staffs and students.

\section{CONCLUSION}

In conclusion, the students and staff can be considered to have high awareness toward OSH as 
the overall mean grade is 4.2. This phenomenon could be attributed to their numerous experiences working in hazardous condition allowing them to gain enough information of OSH. Safety committee is recorded to have the lowest grade which is 3.54 indicating that the students and employees are not very much aware of the committee responsible for the safety in their working environment. Hence, it is highly recommended for the safety committee to provide safety seminars and short courses in regular basis to increase awareness among the staff and students. As comparison between staff and students, it can be concluded that the staffs have higher level of consciousness toward OSH with mean grade of 4.26 as compared to the students (mean grade 4.14). The results could be explained by the years of laboratory experience including handling hazardous chemicals and multiple instrumentations by the staff as compared to the undergraduate students who only conduct common experiments. Circumstances within the university create a complex system that can lead to many challenges. Hence, all the issues that were identified in this study must be structured in order develop a concrete solution and recommendations. Therefore, to ensure that the staff and students meet OSH safety requirements, a few adjustments in leadership committee, risk assessment committee and safety committee are highly recommended.

\section{ACKNOWLEDGMENTS}

This research work is supported by the Project (71372111) supported by NSFC.

\section{REFERENCES}

[1] E. Vilkman, Occupational Safety and Health Aspects of Voice and Speech Professions. Folia Phoniatrica et Logopaedica. 2004. 56(4): 22053.

[2] A. Lay, Morgan The Relationship between Occupational Health and Safety Vulnerability and Workplace Injury. 2017.Safety Science 94:
85-93.

[3] Y. Zuojin, H. Chang Lin, D. Todd, Smith, K. David Lohrmann. Association between Workplace Harassment and Occupational Injury among Adult Workers in the United States. Safety Science . 2018. 110(April): 372 79.

[4] A. El-Gilany, H. Knowledge, Attitude, and Practice (KAP) of 'Teaching Laboratory' Technicians towards Laboratory Safety and Waste Management: A Pilot Interventional Study. Journal of Hospital Infection. 2017. 96(2): 192-94. http://dx.doi.org/10.1016/j.jhin.2017.02.007.

[5] O. Tomasz, M. Snakard. Challenges in Applying Process Safety Management at University Laboratories. Journal of Loss Prevention in the Process Industries .2017. 49: 209-14. http://dx.doi.org/10.1016/j.jlp.2017.06.013.

[6] M. Fred. Reflections of a Former OSHA Official on the Laboratory Standard. Journal of Chemical Health and Safety. 2016.23(5): 6-11.

[7] G. Linda M. The Intervention Research Process in Occupational Safety and Health: An Overview from the National Occupational Research Agenda Intervention Effectiveness Research Team. Journal of Occupational and Environmental Medicine. 2001. 43(7): 61622

[8] F. Ken. OSHA's Lab Standard at 26. Journal of Chemical Health and Safety. 2016. 23(1): 51.

[9] A. Suraiyah, K. Ahsan. Workplace Safety Compliance Implementation Challenges in Apparel Supplier Firms. Journal of Cleaner Production. 2019. 232: 462-73.

[10] P. Jamil, D. Idrus Hadmidah, H. Ashari, F. Zaini Rossilah S. N. Muktar. Level of Awareness of Utm Staff on Occupational. 2004.

[11] A. H. Rosina, C. Yip Hon. Safety Culture and Safety Compliance in Academic Laboratories: A Canadian Perspective. Journal of Chemical Health and Safety. 2018. 25(6): 6-12.

[12] M. Osment, A. Chris. Occupational Safety. Industrial Fabric Products Review. 2002. 87(11): 32-34.

[13] Ahmad, Mohd Fa'iz. 2009. "Tahap Kesedaran Staf UTM Terhadap Keselamatan Pekerjaan Di Makmal Dan Bengkel Kejuruteraan.” Fakulti Pendidikan, Universiti Teknologi Malaysia 6(June): 36-51. 